\title{
PHƯ̛̛NG PHÁP NẮN TRỰC GIAO ẢNH VÊ TINH ĐỘ PHÂN GIẢI CAO SỬ DỤNG MÔ HìNH SỐ Bể MẶT DSM LIDAR
}

\author{
ThS. NGUYỄN TH! THỤC ANH(1) \\ ThS. TĂNG QUÔC CƯƠNG ${ }^{(2)}$ \\ ThS. ĐĂNG THANH TÙNG ${ }^{(3)}$ \\ KS. TRƯƠNG XUÂN QUYÊंN(4)
}

Tóm tắt:

Ngày nay, ảnh viễn thám độ phân giải cao và dữ liệu Lidar đã trở thành hai trong các nguồn dữ liệu chính phục vụ việc xây dựng dữ liệu địa lý độ chính xác cao. Trong các dự án quét Lidar thường chụp ảnh máy bay đồng thời tuy nhiên nếu do điều kiện kỹ thuật (bay quét Lidar đêm hay do trục trặc với máy ảnh) không có ảnh máy bay chụp kèm, ảnh viễn thám độ phân giải cao sẽ là nguồn dữ liệu thay thế tương đối đồng bộ với dữ liệu Lidar về độ chi tiết, có thể kết hợp trong quá trình xử lý cũng như chiết tách thông tin. Việc nắn ảnh viễn thám bằng mô hinh số bề mặt DSM cho phép tăng độ chính xác của ảnh trực giao, khử dịch chuyển do chênh cao của đối tượng với bề mặt DEM đặc biệt là cho khu vực đô thị nơi tập trung dân cư với mật độ nhà cao. Bài báo trình bày kết quả nghiên cứu về các phương pháp nắn ảnh trực giao, yêu cầu về điểm khống chế ảnh, để xuất qui trình nắn ảnh dùng DSM từ Lidar. Kết quả thử nghiệm nắn ảnh WorldView1 độ phân giải $0.5 \mathrm{~m}$ dùng DSM từ dữ liệu Lidar mật độ 2.5 điểm $/ \mathrm{m}^{2}$ cho khu vực thành phố Bắc Giang được phân tích và so sánh với kết quả nắn bằng $D E M$. Kết luận và các khuyến cáo cho việc nắn ảnh vệ tính độ phân giải cao dùng DSM Lidar được đưa ra dựa trên kết quả thử nghiệm này.

\section{Giới thiệu}

$\mathfrak{C}^{2}$ huật ngữ ảnh trực giao - Orthophoto ban đầu được áp dụng cho ảnh hàng không sau đó đến các loại ảnh khác như ảnh mặt đất, ảnh chụp từ vệ tinh. Trên ảnh, các vật thể hầu hết đều chịu ảnh hưởng của hiện tượng dịch chuyển do địa hình relief displacement. Sự dịch chuyển này là kết quả của biến dạng hình học xảy ra khi có các chênh cao trên bề mặt của đối tượng chụp ảnh. (Xem hinh 1)

Để nắn ảnh trực giao cần thiết phải có các dữ liệu sau đây:

1. Ảnh cần nắn;
2. Mô hình số độ cao, trong nắn ảnh trực giao truyền thống đó là mô hình số địa hình DEM của bề mặt mặt đất trần trụi (bare earth); trong nắn ảnh trực giao thực (true orthophotos), mô hình số bề mặt DSM hoặc DEM kết hợp với mô hình nhà - DBM được sử dụng;

3. Các yếu tố định hướng ngoài của ảnh và mô hình số độ cao được cung cấp trong cùng một hệ tọa độ;

4. Các tham số định hướng trong của máy ảnh hay bộ cảm biến.

Các ảnh vệ tinh có độ phân giải cao và siêu cao GSD $\leq 1 \mathrm{~m}$ có thể được sử dụng để

\footnotetext{
(1)Trung tâm Viễn thám Quốc Gia

(2)Tổng cục Biển và Hải đảo,

(3)Trường Đại học Tài nguyên và Môi trường Hà Nội

${ }^{(4)}$ Tổng công ty Tài nguyên và Môi trường
} 
Hình 1: Dịch chuyển do địa hình trên ảnh

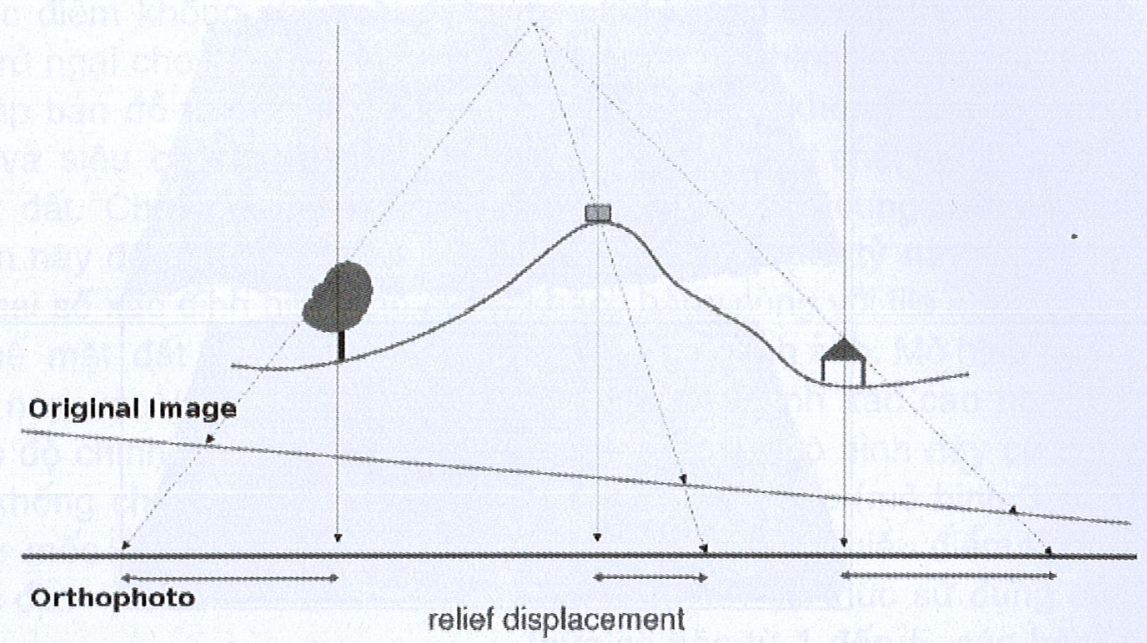

thành lập bản đồ tỷ lệ lớn (chẳng hạn 1/5.000). Tuy nhiên để đạt được độ chính xác của bản đồ ở tỷ lệ này thì ảnh vệ tinh phải được xử lý chính xác qua các công đoạn như định hướng, tham chiếu mặt đất và nắn trực giao. Ở các vùng có chênh cao lớn thì trong nhiều trường hợp, đặc biệt trong các trường hợp mà góc chụp ảnh lệch nhiều so với hướng thiên đế thì yêu cầu phải có DEM hay DSM với độ chính xác cao (với sai số về độ cao không vượt quá $1-2 m$ ). Để đáp ứng được yêu cầu này thì DEM, DSM phải được thành lập bằng các phương pháp như: đo vẽ trực tiếp sử dụng các máy toàn đạc, thủy chuẩn, GPS; đo vẽ lập thể ảnh hàng không và từ những năm gần đây là thành lập từ dữ liệu bay quét LiDAR.

Tại nhiều nước trên thế giới hiện nay các dữ liệu thu được từ công nghệ LiDAR đã trở thành một nguồn dữ liệu chính về thông tin của địa hình. Mặc dù được ứng dụng trong nhiều lĩnh vực khác nhau nhưng mô hình hóa địa hình vẫn là tiêu điểm chính của các công trình, dự án áp dụng công nghệ LiDAR. Việc ứng dụng công nghệ LiDAR trong mô hình hóa địa hình và thành lập mô hình số DEM, DSM với độ chính xác cao (sai số về độ cao nhỏ hơn $0,5 \mathrm{~m}$ ) đã trở nên phổ biến trên thế giới.

Từ năm 2006 đến nay công nghệ bay quét LiDAR cũng đã dần được ứng dụng rộng rãi ở nước ta trong một số dự án như: dự án "Xây dựng cơ sở dữ liệu hệ thống thông tin địa hình - thủy văn cơ bản phục vụ phòng chống lũ lựt và phát triển kinh tế xã hội vùng Đồng bằng sông Cửu Long; dự án: "Thành lập cơ sở dữ liệu nền thông tin địa lý tỷ lệ 1/2.000,1/5.000 cho các khu vực đô thị, khu công nghiệp, khu kinh tế trọng điểm". Công nghệ LiDAR cho phép thành lập DEM, DSM với mức độ chi tiết, mật độ điểm dày và độ chính xác cao (sai số về độ cao có thể đạt tới $5-10 \mathrm{~cm}$ ) hoàn toàn có thể thỏa mãn yêu cầu nắn ảnh trực giao độ phân giải cao và siêu cao cho thành lập bản đồ và các ứng dụng khác ở tỷ lệ $1 / 5.000$ và lớn hơn. (Xem hinh 2)

\section{Phương pháp mô hình hóa trong nắn ảnh vệ tinh}

Mô hình toán học để xử lý ảnh vệ tinh có thể được hiểu là mô hình biểu diễn mối liên kết giữa các phần tử ảnh (pixel) và vị trí chính xác của địa vật có tính tới các sai số 


\section{Jruo đtòi - Ŷ kiến}

Hình 2: Vi dụ về DSM và DEM chiết tách dữ liệu Lidar (nguồn GeoLas Consulting)

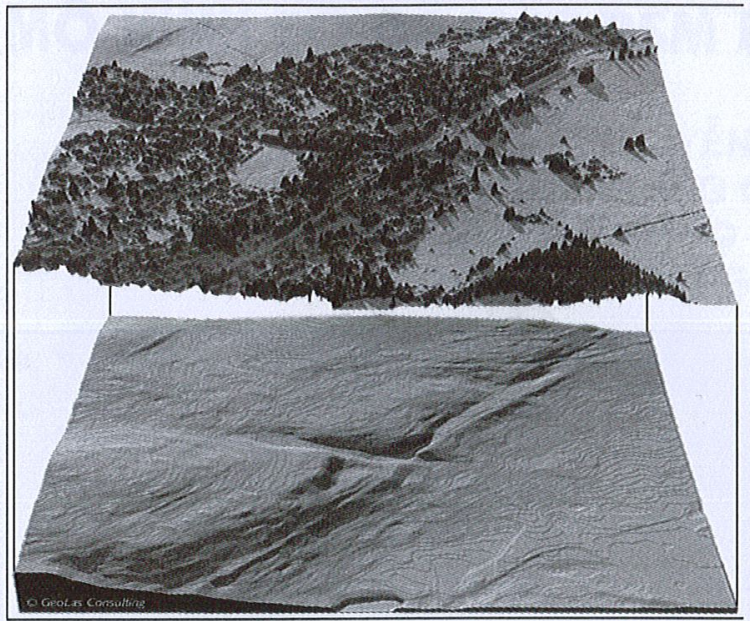

biến dang. Các mô hình toán học được sử dụng để xử lý ảnh vệ tinh (như bình sai tăng dày, thành lập DEM hay nắn trực ảnh) được chia thành hai nhóm: nhóm các mô hình vật lý (physical) hay còn gọi là các mô hình chặt chẽ (rigorous); và nhóm các mô hình tổng quát (generic models). Việc lựa chọn các mô hình này dựa trên mục đích sử dụng và yêu cầu về độ chính xác của sản phẩm, bộ cảm biến (sensor), phần mềm xử lý, dữ liệu ảnh và các tài liệu liên quan được cung cấp, số lượng và phân bố của các điểm khống chế (3)

Mô hình chặt chẽ phản ảnh đúng quá trình tạo ảnh vật lý và các tham số của mô hình này cho biết vị trí và góc nghiêng của bộ cảm biến trong không gian. Mô hình vật lỳ, chẳng hạn như mô hình của phương trình điều kiện đồng tuyến rất thích hợp cho tính toán bình sai tăng dày giải tích cho độ chính xác cao.

Trong mô hình tổng quát các tham số sử dụng để liên kết không gian ảnh và không gian đối tượng không có mối liên quan gì đến tính chất vật lý của bộ cảm biến. Mô hình tổng quát bao gồm các hàm hữu tỷ và các trường hợp đặc biệt của chúng như: mô hình chuyển đổi tuyến tính trực tiếp (DLT); mô hình 3D-affine; mô hình chuyển đổi phép chiếu xuyên tâm (2D projective) và mô hình đa thức $2 \mathrm{D}$ và $3 \mathrm{D}(2 \mathrm{D}$ polynomials, $3 \mathrm{D}$ polynomials). Mô hình tổng quát không phụ thuộc vào loại bộ cảm biến cho nên thích hợp khi sử dụng với các bộ cảm biến khác nhau và khi có bộ cảm biến mới thì các hệ số của mô hình này chỉ cần cập nhật hay tính lại. Tuy nhiên để có thể thay thế được mô hình của bộ cảm biến thì mô hình tổng quát phải gần khớp được với mô hình vật lý.

Việc định hướng ảnh vệ tinh được thực hiện nhờ có các thiết bị định hướng gắn trên vệ tinh như GPS, la bàn con quay hồi chuyển, các bộ cảm đo thiên văn để có được các thông tin về các góc nghiêng của ảnh vệ tinh. Hiện nay việc định hướng trực tiếp ảnh vệ tinh không có điểm khống chế mặt đất có thể đạt độ chính xác cỡ vài mét. Chẳng hạn như với ảnh IKONOS, GeoEye1 hay WorldView1 độ chính xác mặt bằng là vào khoảng từ $3 \mathrm{~m}$ đến $4 \mathrm{~m}(1,2)$. Độ chính xác này là chưa đủ để đáp ứng các công tác thành lập bản đồ tỷ lệ lớn (tỷ lệ 1/5000 hoặc lớn hơn), hơn nữa nếu không có điểm khống chế mặt đất thì việc định hướng trực tiếp nêu trên được thực hiện trong Hệ tọa độ Trắc địa 
toàn cầu WGS-84 chứ không phải là trong các hệ tọa độ quốc gia như VN2000, do đó cần phải có các điểm khống chế mặt đất. Một trong các trở ngại cho việc nắn ảnh vệ tinh và thành lập bản đồ từ ảnh vệ tinh độ phân giải cao và siêu cao là yêu cầu về khống chế mặt đất. Cho hầu hết các loại ảnh vệ tỉnh hiện nay độ chính xác nắn ảnh bị hạn chế bởi sai số xác định hình ảnh của điểm khống chế mặt đất trên ảnh, thông thường sai số này vào khoảng 0,5 pixel. Muốn nâng cao độ chính xác xác định hình ảnh của điểm khống chế mặt đất phải tiến hành đánh dấu mốc cho điểm khống chế mặt đất tại thực địa.

Việc tham chiếu mặt đất chính xác ảnh vệ tinh được thực hiện thông qua quá trình tái tạo mô hình hình học của ảnh. Ngày nay hầu hết các loại ảnh vệ tinh đều được cung cấp cùng với các hệ số của hàm đa thức hữu tỷy, các hệ số này có được dựa trên việc định hướng trực tiếp. Nếu chỉ sử dụng các hệ số này mà không có điểm khống chế mặt đất thì độ chính xác tham chiếu mặt đất đạt được thường không cao, chỉ từ 10 đến $25 \mathrm{~m}$. Để tăng độ chính xác tham chiếu mặt đất của ảnh cần có thêm các điểm khống chế mặt đất. Yêu cầu về số lượng tối thiểu điểm khống chế mặt đất cần sử dụng phụ thuộc vào mô hình toán học được sử dụng. Các mô hình toán học thường được sử dụng để định hướng ảnh vệ tinh là: mô hình quỹ đạo vệ tinh, mô hình hàm hữu tỷ và mô hình hàm đa thức. Sử dụng mô hình này có thể tính toán vị trí và định hướng của bộ cảm biến ở thời điểm chụp ảnh. Không nên sử dụng mô hình quỹ đạo vệ tinh này khi chỉ có một phần của cảnh ảnh gốc, khi cảnh ảnh đã được xử lý hay khi không có các thông tin về quỹ đạo vệ tinh. Mô hình này cho độ chính xác cao nhất. Độ chính xác của mô hình quỹ đạo vệ tinh là khoảng $1 / 3$ pixel cho các ảnh VIR. Mô hình hàm hữu tỷ là mô hình toán học thiết lập mối tương quan giữa điểm ảnh và vị trí của nó trên mặt đất. Nên sử dụng mô hình này khi không có các thông tin cần thiết để xây dựng mô hình quĩ đạo vệ tinh, khi nhà cung cấp ảnh không đưa ra các thông số của mô hình chặt chẽ, khi ảnh đã được xử lý hình học, khi nhà cung cấp ảnh tính toán ra mô hình hàm hữu tỷ này và cung cấp cho khách hàng cùng với file ảnh hoặc khi không có toàn bộ cảnh ảnh. Mô hình hàm hữu tỷ có thể cho độ chính xác cao hơn mô hình đa thức do trong mô hình này có tính đến việc hiệu chỉnh độ cao (mô hình 3D). Tuy nhiên mô hình này cần nhiều điểm khống chế mặt đất. Mô hình đa thức sử dụng các hàm đa thức có bậc từ 1 đến 5 , các hàm này được tính toán dựa trên các điểm khống chế mặt phẳng (mô hình 2D).

\section{Quy trình công nghệ và yêu cầu về điểm khống chế mặt đất}

Quy trình công nghệ nắn ảnh vệ tinh được thể hiên trên hình 2.

\section{Yêu cầu về khống chế mặt đất}

Điểm khống chế ảnh là điểm có thể nhận biết rõ ràng trên ảnh đồng thời có tồn tại trên thực địa. Trong trường hợp có bản đồ tỷ lệ lớn hơn hay bản đồ cùng tỷ lệ đảm bảo độ chính xác thì điểm khống chế ảnh có thể được xác định ngay trên bản đồ đồ. Nếu không có các tài liệu bản đồ đáp ứng các yêu cầu trên thì điểm khống chế ảnh bắt buộc phải được xác định bằng phương pháp đo ngoại nghiệp như đo GPS. Thực tiễn nắn chỉnh hình học ảnh số cho thấy sai số tồn tại tại điểm khống chế ảnh sau bình sai thường nằm trong khoảng 0,3 đến 0,5 pixel. Như vậy có thể thấy rằng sai số đo đạc để xác định ví trí toạ độ của điểm khống chế ảnh trên thực địa không được lớn hơn 0,3 đến 0,5 độ phân giải mặt đất của ảnh số. Ví dụ với ảnh vệ tinh WorldView 1 độ phân giải $0,5 \mathrm{~m}$ cần đo đạc điểm khống chế ảnh ngoại 


\section{Jrao atôi - ý kiến}

Hình 3: Quy trinh công nghệ nắn ảnh vệ tinh độ phân giải cao dùng DSM

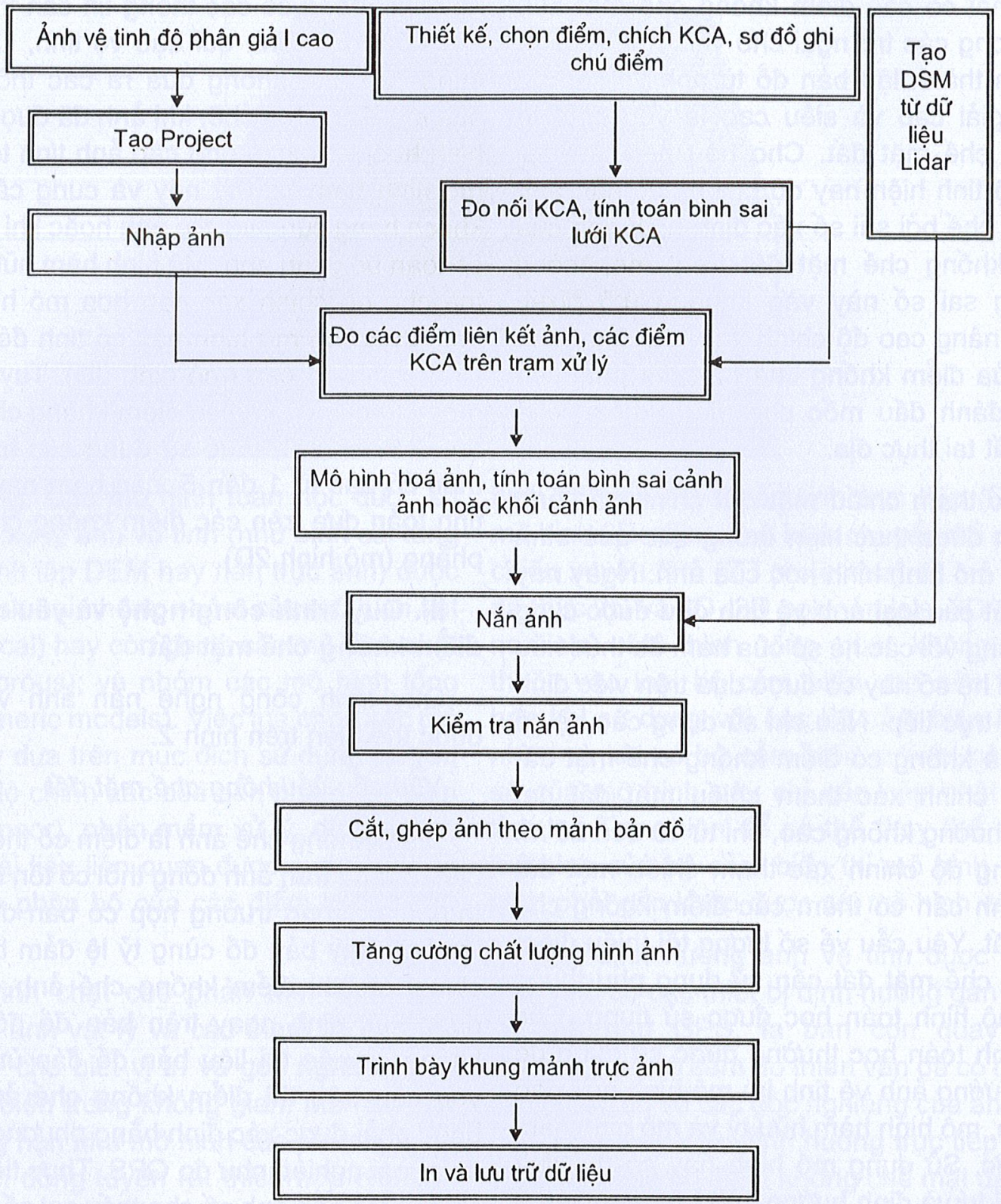

nghiệp với độ chính xác về toạ độ $\mathrm{M}_{\mathrm{XY}} \leq$ $0,1 \mathrm{~m}$. Về độ cao, khoảng cao đều đường bình độ cơ bản trên bản đồ tỷ lệ $1 / 5000$ là $1 \mathrm{~m}$ ở vùng đồng bằng và $5 \mathrm{~m}$ ở vùng đồi, núi. Độ chính xác độ cao của điểm khống chế ảnh không lớn hơn 0,2 khoảng cao đều đường bình độ ở vùng bằng phẳng và 0,3 ở vùng đồi, núi. Tức là, cần đo đạc điểm khống chế ảnh với độ chính xác độ cao là $0,2 \mathrm{~m}$ ở vùng đồng bằng và không lớn hơn $1,5 \mathrm{~m}$ ở vùng đồi núi. (Xem hinh 3)

Số lượng tối thiểu các điểm khống chế ảnh cần có phụ thuộc vào mô hình toán học của ảnh vệ tinh được lựa chọn áp dụng. Chẳng hạn theo lý thuyết số lượng điểm 
khống chế mặt đất tối thiểu cho mô hình đa thức là $n=(t+1) *(t+2) / 2$ trong đó $n$ là số lượng điểm khống chế tối thiểu, $\mathrm{t}$ là bậc của hàm đa thức. Khi áp dụng hàm hữu tỹ với tổng số các hệ số thường được sử dụng là từ 11 (cần it nhất 6 điểm khống chế) đến 29 (cần ít nhất 15 điểm khống chế) theo lý thuyết. Trên thực tế số lượng điểm khống chế ngoại nghiệp cần có luôn nhiều hơn số lượng tính theo lý thuyết để đảm bảo có các trị đo dư và cho phép thực hiện các tính toán, bình sai theo phương pháp bình phương tối thiểu cũng như đánh giá độ chính xác.

Các điểm khống chế ảnh ngoại nghiệp phải được phân bố đều trên toàn bộ cảnh ảnh, trừ trường hợp đặc biệt như: cảnh ảnh chụp ven biển, dọc biên giới hoặc chụp khu vực không thể tiếp cận được... Trong mỗi vùng độ phủ giữa hai cảnh ảnh phải có tối thiểu 3 điểm khống chế. Số lượng điểm kiểm tra trên một cảnh ảnh tối thiểu là 4 điểm, phân bố đều trên toàn bộ cảnh ảnh, và nằm ở khoảng giữa các điểm khống chế ảnh.

IV. Thử nghiệm nắn ảnh vệ tinh độ phân giải cao sử dụng DSM từ dữ liệu Lidar

Khu vực thử nghiệm nằm ở TP Bắc Giang và vùng lân cận. Quá trình thử nghiệm được tiến hành dùng phần mềm ERDAS LPS

\section{Dũ liệu dùng trong thực nghiệm}

Ảnh viễn thám độ phân giải cao được sử dụng trong thực nghiệm này là ảnh WorldView1 độ phân giải $0.5 \mathrm{~m}$.

$D E M$ và $D S M$ dùng trong thử nghiệm này ở dạng Grid trong lưới chiếu VN2000 kinh tuyến trục 105 độ, múi 3 độ được chiết tách tữ dữ liệu điểm Lidar với độ phân giải $0.5 \mathrm{~m}$ sử dụng phần mềm TerraScan. Dữ liệu Lidar này được bay vào thời điểm đầu tháng $11 / 2007$.
Ngoài ra, trong khu vực thử nghiệm có bản đồ địa chính tỷ lệ $1 / 500$ và $1 / 1000$ được đo trong thời gian từ năm 2007 đến 2009. Bản đồ được thành lập trong hệ tọa độ VN2000, múi 3 độ, kinh tuyến trục 107030'.

\section{Quá trình thực nghiệm}

Ảnh WorldView1 được nắn trực giao theo qui trình (hinh 3) sử dụng lần lượt $D E M$ và DSM. Báo cáo chi tiết qui trình nắn, cách phân bố các điểm khống chế và điểm kiểm tra, phương pháp nội suy và tái chia mẫu, và báo cáo về sai số được liệt kê.

Trước khi đưa vào sử dụng, dữ liệu DEM và DSM cũng như bản đồ địa chính được đưa thống nhất về hệ tọa độ VN2000, múi 3 độ, kinh tuyến trục $105^{\circ}$. Đồng thời, dữ liệu DEM và DSM được gộp thành 1 khối duy nhất và chuyển về khuôn dạng IMG để sẵn sàng sử dụng trong phần mềm ERDAS.

Ảnh viễn thám độ phân giải cao được cung cấp không kèm theo thông tin chi tiết về mô hình hình học của bộ cảm biến, hãng cung cấp ảnh đã tính toán xử lý các thông số này trước khi giao ảnh nên mô hình hàm hữu tỷ được chọn để nắn ảnh. Ảnh WorldView1 được lần lượt nắn trực giao bằng gói phần mềm Leica Photogrametry Suite (LPS) của ERDAS sử dụng DEM và DSM từ dữ liệu Lidar. Áp dụng phương pháp nắn ảnh sử dụng mô hình hàm hữu tỷ với 13 điểm khống chế và 4 điểm kiểm tra được phân bố đều trên khu vực ảnh cần nắn. Tọa độ của các điểm khống chế và điểm kiểm tra này được chiết xuất từ bản đồ địa chính tỷ lệ lớn. Sơ đồ phân bố điểm khống chế được thể hiện trên hình 4.

Phương pháp nội suy và tái chia mẫu Resampling method được sử dụng là phương pháp pixel lân cận gần nhất Nearest neighbor với mức độ gối phủ Overlap Threshold 30\%. (Xem hinh 4, bảng 1) 
Hinh 4: Sơ đồ phân bố điểm khống chế và điểm kiểm tra

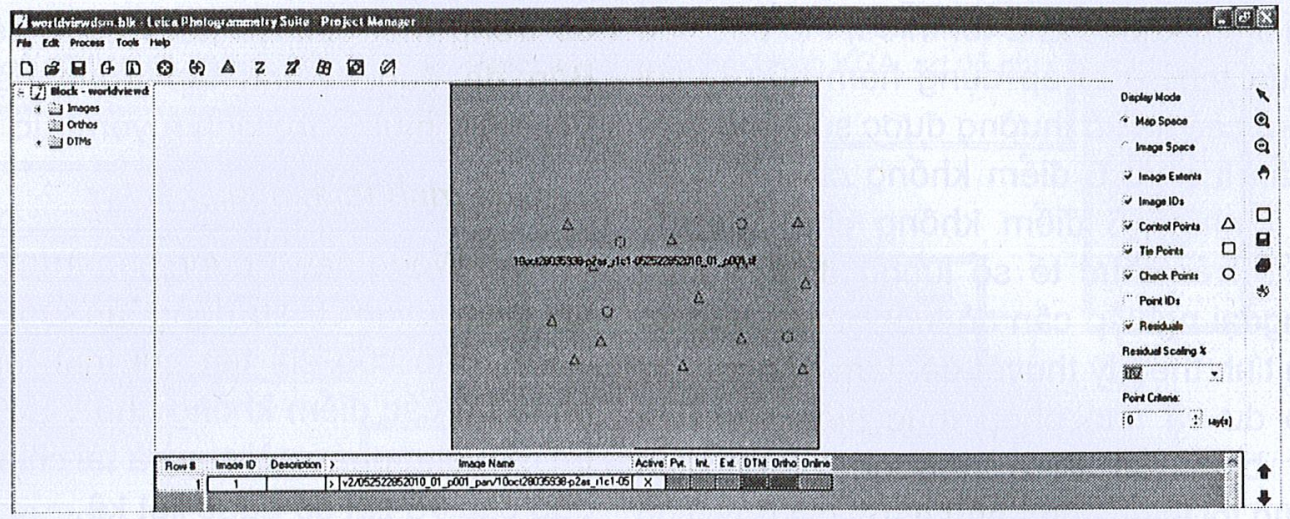

Bảng 1: Bảng thống kê sai số trung phương trong quá trinh nắn (tính bằng pixel) của các điểm khống chế và điểm kiểm tra cho 2 phương pháp nắn

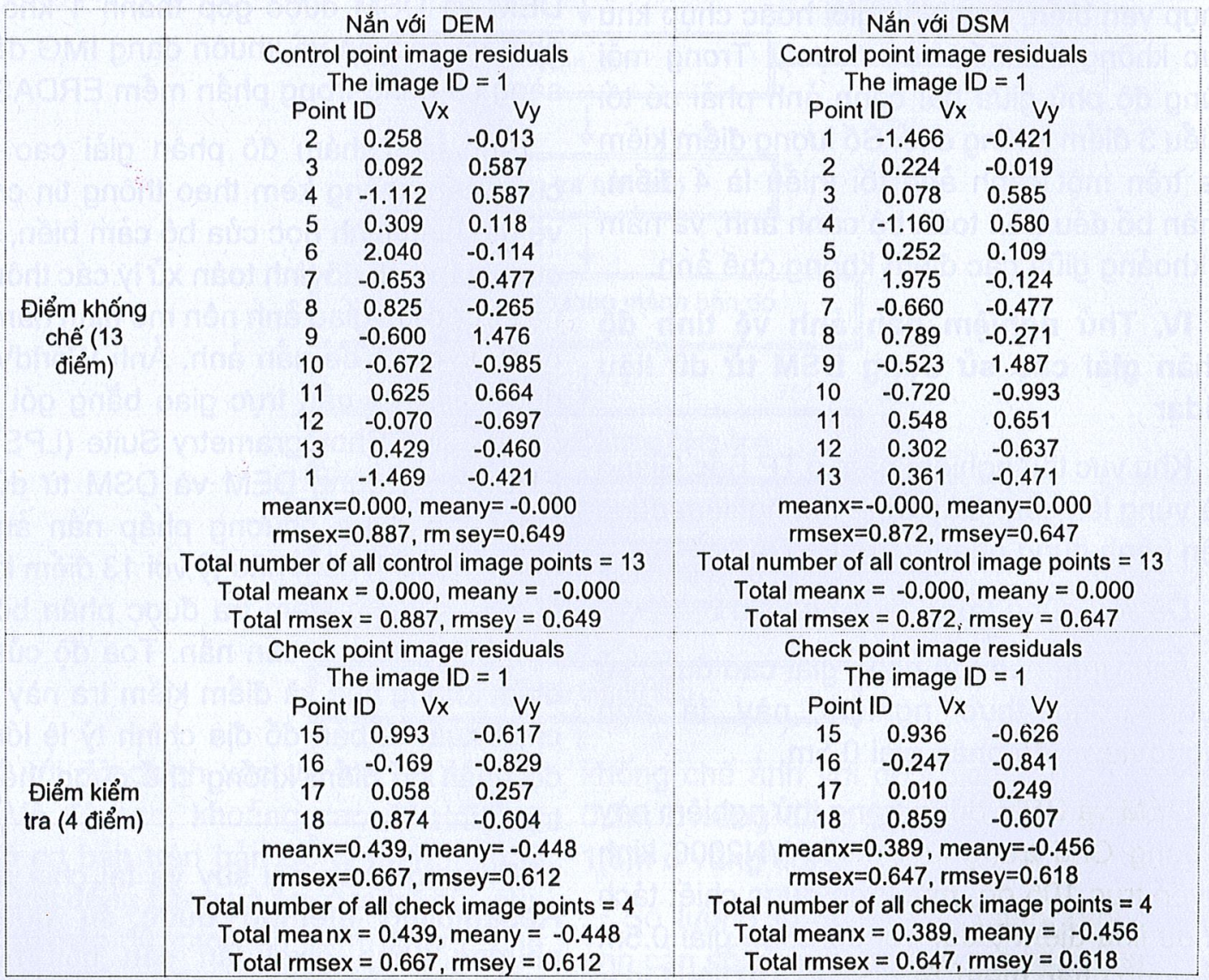


Sai số trung phương vị trí điểm nằm trong khoảng dưới $0.4 \mathrm{~m}$, sai số lớn nhất là $0.9 \mathrm{~m}$. Với độ chính xác này, sản phẩm ảnh trực giao có thể được sử dụng để thành lập bản đồ tỷ lệ 1/5.000 hoặc tốt hơn.

So sánh kết quả nắn trực giao bằng $D E M$ và $D S M$

Kết quả của việc thử nghiệm nắn chuyển này là 2 ảnh của khu vực nghiên cứu, 1 ảnh trực giao - orthophoto được nắn bằng $\mathrm{DEM}$ và 1 "ảnh trực giao gần thực" - Neartrue Orthophoto nắn bằng DSM. Với mục đích so sánh độ dịch chuyển của các đối tượng có độ cao riêng khi nắn ảnh trực giao bằng $D E M$ và bằng $D S M, 100$ điểm kiểm tra đã được xác định và đánh số tương ứng trên cả 2 ảnh. Các điểm này được chọn trên các đối tượng dễ nhận biết và xác định chính xác với các độ cao riêng khác nhau từ các điểm nằm mặt đất như điểm cắt của các đường bờ ruộng nhỏ hay vạch sơn trên sân tennis đến các điểm có độ cao riêng đáng kể như các điểm đỉnh mái nhà, các chi tiết dễ nhận biết trên mái. Tiến hành thống kê tọa độ của các điểm này trên cả 2 ảnh, độ cao riêng của chúng, khoảng dịch chuyển khi nắn bằng
DEM và DSM trên thực tế và khoảng dịch chuyển này theo lý thuyết. (Xem hình 5,6 )

Phân tích kết quả so sánh độ dịch chuyển của đối tượng khi nắn bằng $D E M$ và DSM được thống kê trong phụ lục 1 cho thấy với mức độ chênh cao của đối tượng từ 0 đến $22 \mathrm{~m}$ thì mức độ dich chuyển của điểm cũng biến thiên trong khoảng từ 0 đến $\sim 7 \mathrm{~m}$ cho khu vực thử nghiệm với góc thiên đế xấp xỉ 17 độ. Đồ thị hình 6 thể hiện các độ dịch chuyển theo lý thuyết và trên số liệu thử nghiệm với trục tung là số thứ tự điểm so sánh được sắp xếp tăng dần theo độ cao đối tượng tăng dần. Đường màu đỏ thể hiện mức độ dịch chuyển trên dữ liệu thực nghiệm theo khá sát đường lý thuyết màu xanh với độ lệch dưới $0.5 \mathrm{~m}$ đối với $75 \%$ số điểm, dưới $0.75 \mathrm{~m}$ đối với $90 \%$ số điểm được so sánh, giá trị này nằm trong hạn cho phép và có thể được giải thích bởi khả năng nhận biết và chọn điểm so sánh chỉ ở mức độ chính xác từ $1-2$ pixel $(0,5-1 \cdot 0 \mathrm{~m})$. Có 4 điểm với độ chênh dịch chuyển với lý thuyết $>1 \mathrm{~m}$ được kiểm tra lại và sai số lớn này là do sự thay đổi của thực địa trong khoảng thời gian lệch đáng kể giữa ảnh viễn thám (10/2010) và dữ liệu DEM, DSM (11/2007).

Hinh 5: Xác định độ lệch điểm trên ảnh trực giao nắn bằng DEM và bằng DSM Điểm màu đỏ: Vị trí đối tượng trên ảnh nắn bằng DEM Điểm màu vàng tương ứng: Vị trí điểm trên ảnh nắn bằng $D S M$

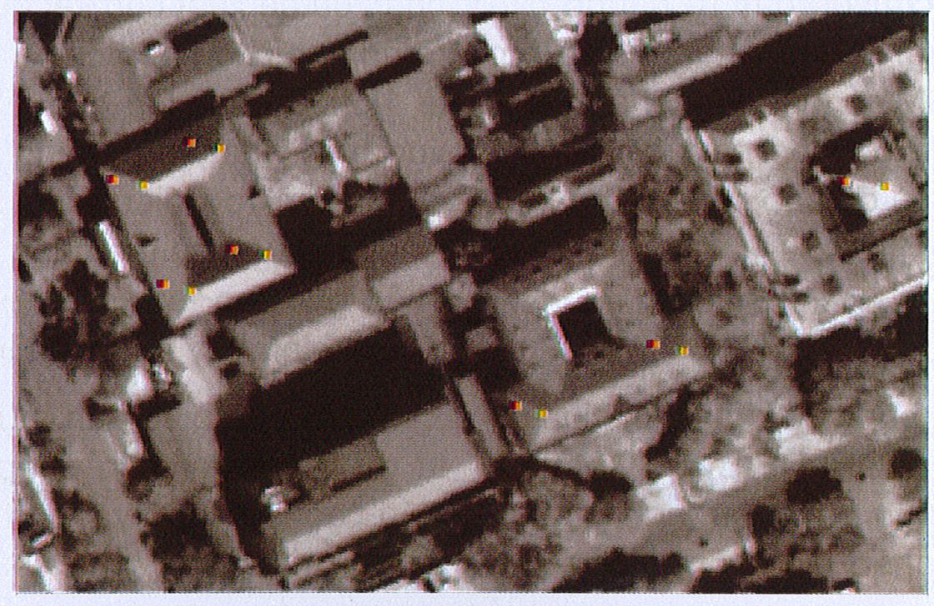


Hinh 6: Đồ thị tương quan độ dịch chuyển vs. độ cao đối tượng

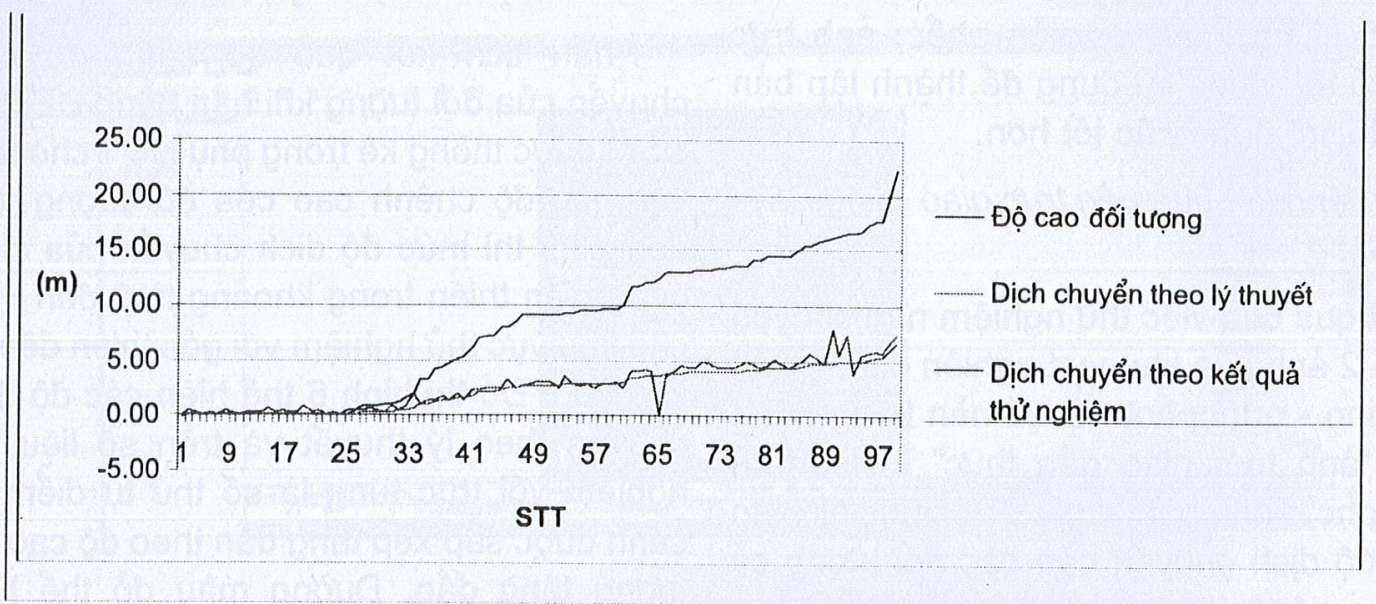

\section{Đánh giá chung}

Ảnh WorldView1 được dùng cho thử nghiệm được chụp trong quĩ đạo bay của vệ tinh từ phía bắc xuống phía nam với góc thiên đế hầu như không đổi cho toàn khu vực thử nghiệm là 17.6 độ. Kết quả so sánh ảnh trực giao nắn bằng $\mathrm{DEM}$ và bằng $\mathrm{DSM}$ cho thấy các đối tượng có độ cao riêng đáng kể đều có hướng đổ tương đối đồng nhất là đông đông nam. Hướng đổ và mức độ dịch chuyển của đối tượng có chiều cao đáng kể đối với hướng bay của vệ tinh cho kết quả sát với các giá trị tính toán theo lý thuyết.

Kết quả nắn ảnh trực giao sử dụng DSM từ dữ liệu Lidar so với sử dụng DEM đã khử được sai số dịch chuyển gây ra bởi độ cao riêng của đối tượng. Tuy nhiên trên ảnh trực giao nắn bằng DSM có hiện tượng "ảnh ma" - Ghost image. Hiện tượng này đặc biệt rõ với các đối tượng có độ cao riêng đáng kể.

Ngoài ra, ở viền mép của mái nhà cũng xuất hiện các hiện tượng răng cưa làm giảm độ mịn của ảnh ở các vị trí này. Hiện tượng này được giả thích bằng sự khác biệt giữa độ phân giải của ảnh WORLDVIEW1 $(0.5 \mathrm{~m})$ và DSM. Tuy DSM dùng trong thử nghiệm này là dạng Grid, độ phân giải $0.5 \mathrm{~m}$ giống như độ phân giải của ảnh WORLDVIEW1 nhưng dữ liệu gốc để tạo DSM là điểm Lidar với khoảng cách giữa các điểm trung bình khoảng $0.8 \mathrm{~m}$. Với mật độ điểm này, mô hình DSM có thể bao gồm các sai số mặt phẳng lên tới $1-1.5 \mathrm{~m}$ khi lột tả các yếu tố có chênh cao lớn như mái nhà và do đó gây ra hiện tượng trượt pixel khi nắn ảnh trực giao nên ảnh trực giao nắn bằng $\mathrm{DSM}$ này không có hình ảnh mịn dọc theo các đường viền mái nhà như khi nắn bằng $\mathrm{DEM}$ mà hơi răng cưa.

\section{Kết luận}

* Ảnh vũ trụ độ phân giải cao nắn trực giao gần thực dùng DSM Lidar theo phương pháp đã đề xuất cho kết quả tốt. Sai số nằm trong hạn cho phép cho thành lập bản đồ tỷ lệ 1/5000 hoặc lớn hơn. Đối với những khu vực bay Lidar mà không có ảnh máy bay chụp kèm (do điều kiện thời tiết hay điều kiện kỹ thuật) có thể dùng ảnh viễn thám độ phân giải cao thay thế trong thành lập bản đồ địa hình hay cơ sở dữ liệu GIS tỷ lệ lớn.

* Ảnh trực giao gần thực nắn bằng DSM từ dữ liệu Lidar cho phép xác định đường viền các khối nhà và vị trí các đối tượng có độ cao riêng lớn (như cầu vượt) chính xác 


\section{Juno đtỏi - Ý kiến}

hơn so với ảnh trực giao nắn bằng $D E M$ thông thường.

* Để có kết quả tốt cần có nhiều ảnh với các góc chụp khác nhau để có ảnh bù cho các đối tượng có độ cao riêng lớn, bị ảnh hưởng bởi hiện tượng "ảnh ma". Tuy nhiên để có ảnh chụp 2 hay nhiều góc chụp khác nhau của cùng 1 khu vực cần có khảo sát kỹ về các ảnh có sẵn trong thư viện và lên kế hoạch đặt ảnh chi tiết với yêu cầu về góc chụp và chiều chuyển động trên quĩ đạo của vệ tinh.

* Để giảm hiện tượng răng cưa tại viền mái các khối nhà trên ảnh trực giao gần thực nên dùng DSM độ phân giải cao được tạo từ dữ liệu Lidar có mật độ điểm cao hơn.

* Để tránh các sai số cục bộ tại các điểm có thay đổi trên thực địa trong khoảng thời gian giữa thời điểm chụp ảnh viễn thám độ phân giải cao và thời điểm bay Lidar nên chọn DSM Lidar và ảnh vệ tinh độ phân giải cao có cách biệt về thời gian nhỏ. $O$

\section{TÀl LIẸU THAM KHẢO}

[1]. Jacobsen, K. 2011 Images of Change, Geospatial World, pp 60-64.

[2]. Jacobsen, K. 2009 Very high resolution satellite images - competition to aerial images, Map World Forum Hyderabad. India.

[3]. Samadzadegan, F., Milanlak, A. and Majdabadi, M. 2006. Geometrical Corrections of Satellite Images by Generic Models, Proceedings of "Remote Sensing: From Pixels to Processes", Enschede, the Netherlands.

[4]. US Army Corps of Engineers. 2003. Remote Sensing, Engineer Manual, 127p.O

\section{Summary}

\section{ORTHO-RECTIFICATION OF HIGH RESOLUTION SATELLITE IMAGE UTILIZING LIDAR DSM}

MSc. Nguyen Thuc Anh

MSc. Tang Quoc Cuong

MSc. Dang Thanh Tung

\section{Eng. Truong Xuan Quyen}

Nowadays, high resolution satellite image and Lidar data are two of the main data source for setting up high accuracy detailed GIS. In Lidar project, airphoto usually acquired simultaneously but if due to technical situation (night fly or malfunction with photocamera) there is no airphoto, high resolution satellite image is relatively compatible replacement in term of level of detail to accompany Lidar data through post-processing as well as information extraction. The ortho- rectification using DSM increase accuracy of orthophoto, remove displacement of high feature especially for residential area with high density of building. This article present research results on methods for satellite image rectification, requirements of control points and proposed the a workflow for ortho-rectification of satellite image using DSM Lidar. Experimental work in the town of BacGiang with WorldView1, panchromatic, resolution $0.5 \mathrm{~m}$ image utilizing DSM from Lidar 2.5 points $/ \mathrm{m}^{2}$ is introduced. Results of ortho-rectification using DEM and DSM are compares and evaluated. Conclusions and recommendations for applying proposed workflow in the future drawn from experimental results. $\mathrm{O}$ 